\title{
IMPROVED MACHINE LEARNING FOR IMAGE CATEGORY RECOGNITION BY LOCAL COLOR CONSTANCY
}

\author{
Hamid Reza Vaezi Joze, Mark S. Drew \\ School of Computing Science, Simon Fraser University \\ Vancouver, BC, Canada V5A 1S6 \\ $\{$ hrv1,mark\}@cs.sfu.ca
}

\begin{abstract}
Color constancy is the ability to recognize colors of objects invariant to the color of the light source. Systems for object detection or recognition in images use machine learning based on image descriptors to distinguish object and scene categories. However, there can be large variations in viewing and lighting conditions for real-world scenes, complicating the characteristics of images and consequently the image category recognition task. To reduce the effect of such variations, either color constancy algorithms or illuminationinvariant color descriptors could be used.

In this paper, we evaluate the performance of straightforward color constancy methods in practice, with respect to their utilization in a standard object classification problem, and also investigate their effects using local versions of these algorithms. These methods are then compared with color invariant descriptors. In a novel contribution, we ascertain that a combination of local color constancy methods and color invariant descriptors improve the performance of object recognition by as much as more than 10 percent, a significant improvement.
\end{abstract}

Index Terms- Local Color Constancy, Category Recognition, Bag of Words.

\section{INTRODUCTION}

Color constancy is the ability to recognize colors of objects invariant to the color of the light source. The simplest color constancy method, the so-called max-RGB method, estimates the light source color from the maximum response of each of the RGB color channels [1]. Another well-known color constancy method is based on the gray-world hypothesis [2], which assumes that the average reflectance in the scene is achromatic. Gray-edge is a recent new version of the grayworld hypothesis that says: the average of the reflectance differences in a scene is achromatic [3]. Although more elaborate algorithms exist, methods such as gray-world, gray-edge and max-RGB are still widely used because of their low computational costs and quite reasonable performance.
Many systems for object detection or recognition in images use machine learning based on image descriptors to distinguish object and scene categories. However, there can be large variations in viewing and lighting conditions for realworld scenes, complicating the characteristics of images and consequently the image category recognition task. To reduce the effect of such variations, either color constancy algorithms or illumination-invariant color descriptors could be used.

Burghouts and Geusebroek [4] and van de Sande et al. [5] evaluated the performance of (invariant) local color descriptors for the classification of object categorization. Here we evaluate the effect of color constancy on the performance of color descriptors in the object classification problem and compare them to local color invariant descriptors.

In this paper, we evaluate the performance of straightforward color constancy methods in practice, with respect to their utilization in a standard object classification problem. They are compared with existing color invariant descriptors, which usually try to omit to some degree the effect of illumination conditions. For this purpose the SIFT descriptor [6] in three color channels (RGB-SIFT) is selected for use because of its good performance in the object classification problem. We carry out the learning process using the standard bag-of-words method over image sets, preprocessing using color constancy algorithms, and compare the performance of these methods with other color descriptors such as C-SIFT and Opponent-SIFT [4]. We show that applying color constancy algorithms locally outperforms the global use of these algorithms in the object recognition problem. We determine that a combination of (1) these local color constancy methods and (2) local color invariant descriptors improves the performance of object recognition substantially, by as much as more than 10 percent, a significant improvement.

In summary, we utilize a combination of these methods in the learning phase, applying multiple kernel learning by adding the kernel function of all methods. We also suggest combining single color invariant descriptor and local color constancy methods to reduce the cost complexity of combination method while keeping the precision not far from the best result which is combining all methods by multiple kernel 
learning.

The paper is organized as follows. In $\S 2$ color constancy methods that we use in this paper are briefly described. In $\S 3$ we investigate the result of object classification using color constant images. Section 4 contains combination methods, and shows us which combination of methods give us the best result. Section 5 concludes the paper with some observations on the method proposed.

\section{COLOR CONSTANCY}

Several color constancy methods have been proposed. The color diagonal transform method is the simplest and was shown [7] to have good results, when utilized with a spectral sharpening color space transform, notwithstanding its simplicity. Typically, however, a diagonal method is applied in practice by simply multiplying each untransformed color channel by a single value.

The first color constancy method, the so-called max-RGB method, estimates the light source color from the maximum response of the different color channels [1]. Another wellknown color constancy method is based on the gray-world hypothesis [2], which assumes that the average reflectance in the scene is achromatic. Finlayson and Trezzi [8] formalize these two methods by subsuming them into a single formula using the Minkowski norm:

$$
\left(\frac{\int f^{p}(x) d x}{\int d x}\right)^{\frac{1}{p}}=k e
$$

where $e$ is light source color and $k$ is a scaling constant. For $p=1$ the equation is equal to the gray-world assumption and for $p=\infty$ it is equal to color constancy by max-RGB, which is based on the assumption that the maximum response in the channels is caused by a white patch.

Gray-edge is a recent version of the gray-world hypothesis that says: the average of the reflectance differences in a scene is achromatic [3]. Using the same formulation we make use of the gradient image:

$$
\left(\frac{\int\left|f_{x}(x)\right|^{p} d x}{\int d x}\right)^{\frac{1}{p}}=k e
$$

The simplest Gray-edge is for $p=1$. Since we can find $e$ by these methods we can then enter $e$ into a diagonal transform matrix to apply to the image. Although more elaborate algorithms exist, methods such as gray-world, gray-edge and max-RGB are widely used because of their low computational cost and quite adequate performance. Figs. 1(b,c,d) show the result of color constancy by the mentioned global methods.

However in natural scene images, the light source is not constant over the whole image. But we could nevertheless assume that the light source is constant for small regions of an image and locally apply a diagonal transform matrix for each

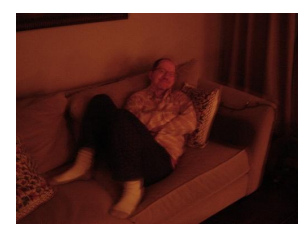

(a)

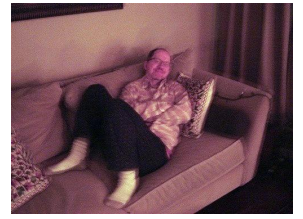

(b)

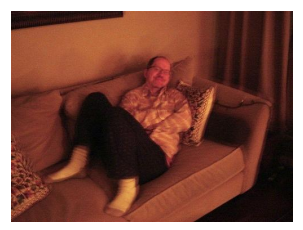

(d)

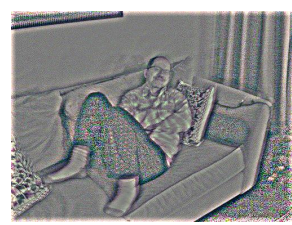

(f)

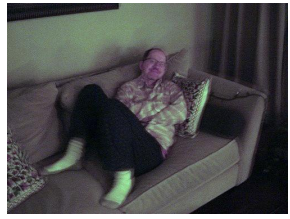

(c)

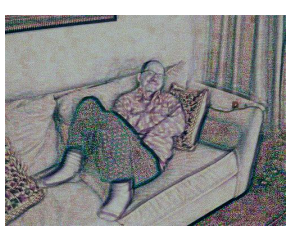

(e)

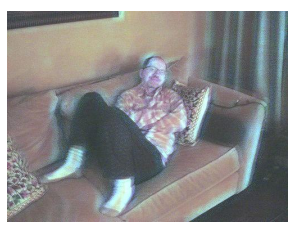

(g)
Fig. 1. (a,b,c,d) Input image and the result of color constancy methods: max-RGB, gray-world and gray-edge. $(\mathrm{e}, \mathrm{f}, \mathrm{g})$ Local color constancy: results of color constancy methods maxRGB, gray-world and gray-edge for each small region of the image.

small region of the image rather than the whole image. Let us denote this scheme as "local color constancy". Figs. 1(e,f,g) show the result for local color constancy by the mentioned methods.

In the next section we show the object classification process over color constant images instead of input images, so as to discover the effect of illumination in the learning process.

\section{LEARNING BY BAG OF WORDS}

We now apply color constancy methods to the object categorization problem. For this purpose the SIFT descriptor [6] in three color channels (RGB-SIFT) is selected for use because of its good performance in the target application.

The Bag-of-Words [9] model is adopted as the learning process here because of its demonstrated high performance in object recognition. Codebooks for bag-of-words methods are constructed by clustering the features of points from a set of training images using a method such as k-means. Our code- 
book size is 4000. The bag-of-words model performs vector quantization of the color descriptors in an image against a visual codebook. A descriptor is assigned to the codebook element which is closest in Euclidean space. We use the reference implementation provided by [5].

For learning concept classifiers, the Support Vector Machine (SVM) algorithm is used by all state-of-the-art systems. Hence we use SVM to classify the object categories by the following kernel function, where the SVM kernel is based on the $\chi^{2}$ distance between two features:

$$
k\left(F_{1}, F_{2}\right)=e^{-\frac{1}{D} d i s t_{\chi^{2}}\left(F_{1}, F_{2}\right)}
$$

where $F_{1}$ and $F_{2}$ are feature vectors. We do not apply a spatial pyramid, which includes spatial information into the bag-ofwords model, so as to be more sensitive to the effect of color constancy methods. (A $2 \%$ improvement has been found in [5] for C-SIFT or Opponent-SIFT by applying a spatial pyramid.)

As data, we use The PASCAL VOC Challenge 2007 dataset which contains nearly 10,000 images of 20 different object categories, e.g. bird, bottle, car, dining table, motorbike and people. The dataset is divided into a predefined training set (5011 images) and test set (4952 images).

The result of object categorization for all 20 objects in terms of mean average precision for the three color constancy methods and their local version is presented in Fig. 2. The average precision is taken as the performance metric for determining the accuracy of ranked category recognition results which is a single-valued measure that is proportional to the area under a precision-recall curve, so it combines both precision and recall into a single performance value. We also present the result of invariant color descriptors C-SIFT and Opponent-SIFT (since they were reported as best invariant color descriptors in [4] and [5]), as well as the normal SIFT, in comparison to RGB-SIFT over color constant images.

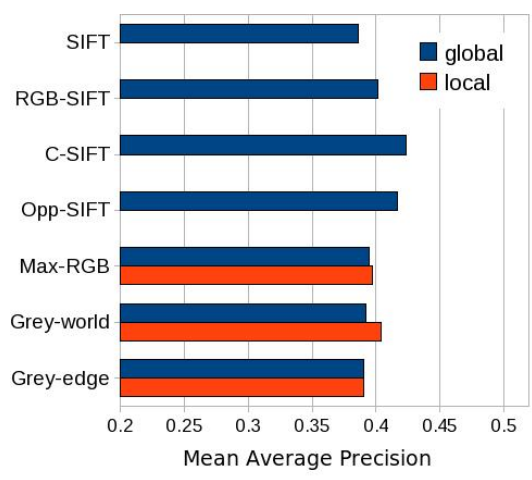

Fig. 2. The result of object categorization for all 20 objects in terms of mean precision for 3 color constancy method and their local version compared to invariant color descriptors CSIFT and Opponent-SIFT, and normal SIFT.

We can see that invariant color descriptors (C-SIFT and
Opponent-SIFT) outperform methods with color constancy preprocessing. But on the other hand, use of color constancy methods locally achieves better results in comparison with their global usage.

Fig. 3 illustrates the average precision for each object category when we apply bag-of-words for image sets, preprocessing each of the color constancy methods of the previous section besides using RGB-SIFT, C-SIFT and OpponentSIFT descriptor.

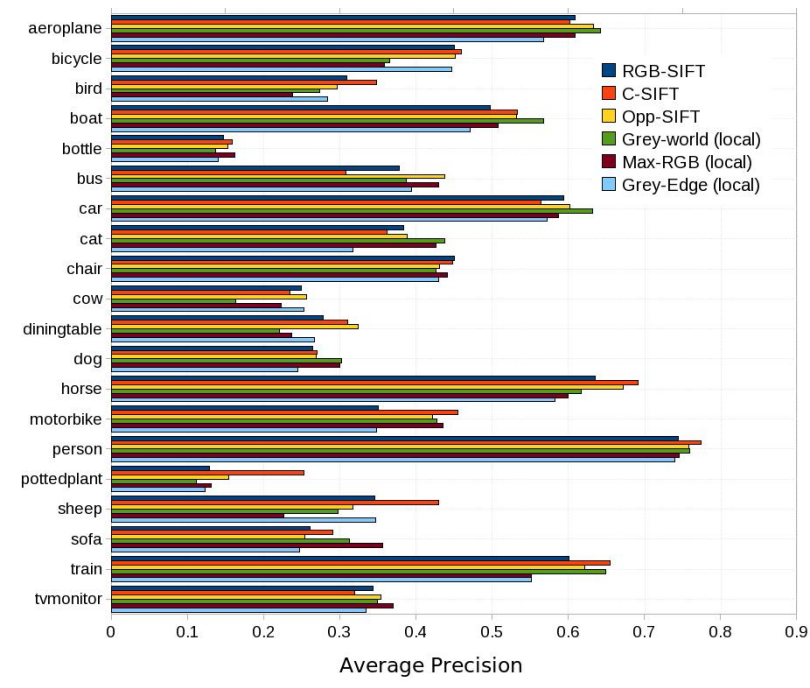

Fig. 3. The average precision for each object category when we apply bag-of-words for each color constancy method besides C-SIFT descriptors.

C-SIFT is best overall, but not in all categories. Hence the question: can we combine features for a better result?

\section{COMBINATION}

Comparing the result for each category in Fig. 3 , it is clear that although C-SIFT has the best mean average precision, no method is best for all categories. A straightforward method to get a better result is to apply multiple kernel learning [10] to linearly add kernel functions for each of the features to reach a new kernel that has the best result from each of them. Using the 6 kernels used in Fig. 3, multiple kernel learning give us a mean average precision of 0.523 , which is a 10 percent improvement over C-SIFT that is the best single color invariant descriptor.

But, using all the kernels is time-consuming, so we are interested in finding fewer kernels which have results near multiple kernel learning's result. In Table 1 we compute the result over any two kernels to see which of them have redundant information. Suppose $K_{M_{1}}$ and $K_{M_{2}}$ are kernel functions for methods $M_{1}$ and $M_{2}$. We can compute the combination ker- 
Table 1. The result in terms of mean average precision over any two kernels.

\begin{tabular}{|c|c|c|c|c|c|}
\hline & 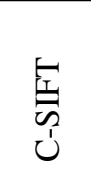 & 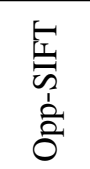 & 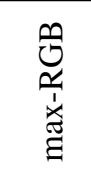 & 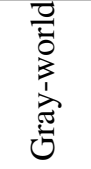 & 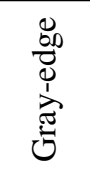 \\
\hline C-SIFT & 0.423 & 0.477 & 0.479 & 0.491 & 0.473 \\
\hline Opp-SIFT & - & 0.416 & 0.469 & 0.479 & 0.452 \\
\hline max-RGB & - & - & 0.397 & 0.454 & 0.455 \\
\hline Gray-world & - & - & - & 0.404 & 0.466 \\
\hline Gray-edge & - & - & - & - & 0.390 \\
\hline
\end{tabular}

nel function for feature vectors $F_{1}$ and $F_{2}$ and $F_{1}^{\prime}$ and $F_{2}^{\prime}$ as

$$
k_{M_{1} M_{2}}\left(F_{1}, F_{2}, F_{1}^{\prime}, F_{2}^{\prime}\right)=e^{\ln \left(K_{M_{1}}\left(F_{1}, F_{2}\right)\right)+\ln \left(K_{M_{2}}\left(F_{1}^{\prime}, F_{2}^{\prime}\right)\right)}
$$

Using the individual kernel function in eq. 3, we have:

$$
k_{M_{1} M_{2}}\left(F_{1}, F_{2}, F_{1}^{\prime}, F_{2}^{\prime}\right)=e^{-\frac{1}{D} d i s t_{\chi^{2}}\left(F_{1}, F_{2}\right)-\frac{1}{D^{\prime}} d i s t_{\chi^{2}}\left(F_{1}^{\prime}, F_{2}^{\prime}\right)}
$$

Table 1 has some interesting points. First of all, using just two kernels, C-SIFT and local Gray-world, we have mean average precision of 0.491 that is significant while reaching this degree of precision is quite inexpensive compared to multiple kernel learning. On the other hand, the table shows that although color constancy methods do not have good results compared to color invariant descriptors such as C-SIFT and Opponent-SIFT, when we combine them with any of invariant color descriptors the result is better compared to combining the different color invariant descriptors by themselves. That means these color constancy methods have some information which is not covered by color invariant descriptors. Figure 4 compares the result of combining all methods by multiple kernel learning (MKL), combining C-SIFT and local gray-world kernels by eq. 5 and the best invariant color descriptors.

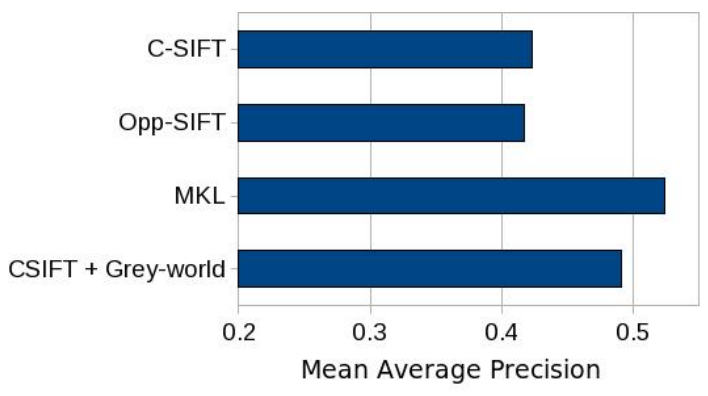

Fig. 4. Final comparison

\section{CONCLUSION}

In this paper, we evaluate the performance of straightforward color constancy methods in practice, with respect to their uti- lization in a standard object classification problem (The PASCAL VOC Challenge 2007 dataset). They are compared with existing color invariant descriptors, which usually try to omit to some degree the effect of illumination conditions. We show that applying color constancy algorithms locally outperforms the global use of these algorithms in the object recognition problem. We determine that a combination of (1) these local color constancy methods and (2) local color invariant descriptors by multiple kernel learning improves the performance of object recognition substantially, by as much as more than 10 percent, a significant improvement. We also suggest combining C-SIFT and the local Gray-world method to reduce the cost complexity of a combination method while retaining a precision not far from best result, which is combining all methods by multiple kernel learning.

Finally, We show that although the simple color constancy methods as a preprocessor to color descriptors for learning process do not achieve better performance by themselves, combining these features with color invariant descriptors increases performance significantly, implying that using these color constancy methods we usually have some information which is not included in color invariant descriptors.

\section{REFERENCES}

[1] K. Barnard, V. Cardei, and B.V. Funt, "A comparison of computational color constancy algorithms-part i: Methodology and experiments with synthesized data," in IEEE Trans. on Image Proc., 2002, vol. 11(9), pp. 972-984.

[2] G. Buchsbaum, "A spatial processor model for object colour perception," J. Franklin Inst., vol. 310, pp. 1-26, 1980.

[3] J. van de Weijer and T. Gevers, "Color constancy based on the grey-edge hypothesis," in Int. Conf. on Image Proc., 2005, pp. II:722-725.

[4] G. J. Burghouts and J. M. Geusebroek, "Performance evaluation of local colour invariants," in Comput. Vision Image Understanding, 2005, vol. 113, pp. 48-62.

[5] K. E. A. van de Sande, T. Gevers, and C. G. M. Snoek, "Evaluating color descriptors for object and scene recognition," in IEEE Trans. on Patt. Anal. and Machine Intell., 2010.

[6] D. G. Lowe, "Distinctive image features from scale-invariant keypoints," in Int. J. of Comput. Vision, 2004, vol. 60, pp. 91110.

[7] G. D. Finlayson, M. S. Drew, and B. V. Funt, "Diagonal transforms suffice for color constancy," in Int. Conf. on Comput. Vision, 1993, pp. 164-171.

[8] G.D. Finlayson and E. Trezzi, "Shades of gray and colour constancy," in Twelfth Color Imageing Conference: Color, Science, Systems and Applications., 2004, pp. 37-41.

[9] F. Jurie and B. Triggs, "Creating efficient codebooks for visual recognition," in Int. Conf. on Comput. Vision, 2005, pp. 15311565.

[10] G. Rätsch, S. Sonnenburg, and C. Schäfer, "Large scale multiple kernel learning," J. of Machine Learning Res., vol. 7, pp. 1531-1565, 2006. 